\title{
OLD AND NEW EXAMPLES OF SCALE FUNCTIONS FOR SPECTRALLY NEGATIVE LÉVY PROCESSES
}

\author{
F. HUBALEK, ${ }^{*}$ Vienna University of Technology
}

A. E. KYPRIANOU, ${ }^{* *}$ The University of Bath

\begin{abstract}
We give a review of the state of the art with regard to the theory of scale functions for spectrally negative Lévy processes. From this we introduce a general method for generating new families of scale functions. Using this method we introduce a new family of scale functions belonging to the Gaussian Tempered Stable Convolution (GTSC) class. We give particular emphasis to special cases as well as cross-referencing their analytical behaviour against known general considerations.
\end{abstract}

Keywords: Scale functions, Spectrally negative Lévy processes, Mittag-Leffler functions, Wiener-Hopf factorization

2000 Mathematics Subject Classification: Primary 60G51, 60J75

Secondary 60G99

\section{Spectrally negative Lévy processes and scale functions}

Let $X=\left\{X_{t}: t \geq 0\right\}$ be a Lévy process defined on a filtered probability space $(\Omega, \mathcal{F}, \mathbb{F}, \mathbb{P})$, where $\left\{\mathcal{F}_{t}: t \geq 0\right\}$ is the filtration generated by $X$ satisfying the usual conditions. For $x \in \mathbb{R}$ denote by $\mathbb{P}_{x}$ the law of $X$ when it is started at $x$ and write simply $\mathbb{P}_{0}=\mathbb{P}$. Accordingly we shall write $\mathbb{E}_{x}$ and $\mathbb{E}$ for the associated expectation operators. In this paper we shall assume throughout that $X$ is spectrally negative meaning here that it has no positive jumps and that it is not the negative of a subordinator. It is well known that the latter allows us to talk about the Laplace exponent $\psi(\theta):=\log \mathbb{E}\left[e^{\theta X_{1}}\right]$ for $\Re(\theta) \geq 0$ where in particular we have the Lévy-Khintchine representation

$$
\psi(\theta)=-a \theta+\frac{1}{2} \sigma^{2} \theta^{2}+\int_{(-\infty, 0)}\left(e^{\theta x}-1-x \theta \mathbf{1}_{\{x>-1\}}\right) \Pi(d x)
$$

where $a \in \mathbb{R}, \sigma \geq 0$ is the Gaussian coefficient and $\Pi$ is a measure concentrated on $(-\infty, 0)$ satisfying $\int_{(-\infty, 0)}\left(1 \wedge x^{2}\right) \Pi(d x)<\infty$. The, so-called, Lévy triple $(a, \sigma, \Pi)$ completely characterises the process $X$.

For later reference we also introduce the function $\Phi:[0, \infty) \rightarrow[0, \infty)$ as the right inverse of $\psi$ on $(0, \infty)$ so that for all $q \geq 0$

$$
\Phi(q)=\sup \{\theta \geq 0: \psi(\theta)=q\} .
$$

\footnotetext{
** Postal address: Vienna University of Technology, Financial and Actuarial Mathematics, Wiedner Hauptstraße 8/105-1, A-1040 Vienna, Austria. email: fhubalek@fam.tuwien.ac.at

* Postal address: Department of Mathematical Sciences, The University of Bath, Claverton Down, Bath BA2 7AY. email: a.kyprianou@bath.ac.uk
} 
Note that it is straightforward to show that $\psi$ is a strictly convex function which is zero at the origin and tends to infinity at infinity and hence there are at most two solutions of the equation $\psi(\theta)=q$.

Suppose now we define the stopping times for each $x \in \mathbb{R}$

$$
\tau_{x}^{+}=\inf \left\{t>0: X_{t}>x\right\} \text { and } \tau_{x}^{-}=\inf \left\{t>0: X_{t}<x\right\} .
$$

A fluctuation identity with a long history concerns the probability that $X$ exits an interval $[0, a]$ (where $a>0$ ) into $(a, \infty)$ before exiting into $(-\infty, 0)$ when issued at $x \in[0, a]$. In particular it is known that

$$
\mathbb{E}_{x}\left(e^{-q \tau_{a}^{+}} \mathbf{1}_{\left\{\tau_{a}^{+}<\tau_{0}^{-}\right\}}\right)=\frac{W^{(q)}(x)}{W^{(q)}(a)}
$$

where $x \in(-\infty, a], q \geq 0$ and the function $W^{(q)}: \mathbb{R} \rightarrow[0, \infty)$ is defined up to a multiplicative constant as follows. On $(-\infty, 0)$ we have $W^{(q)}(x)=0$ and otherwise $W^{(q)}$ is a continuous function (right continuous at the origin) with Laplace transform

$$
\int_{0}^{\infty} e^{-\theta x} W^{(q)}(x) d x=\frac{1}{\psi(\theta)-q} \text { for } \Re(\theta)>\Phi(q) .
$$

The functions $\left\{W^{(q)}: q \geq 0\right\}$ are known as scale functions and for convenience and consistency with existing literature we write $W$ in place of $W^{(0)}$. Identity (4) exemplifies the relation between scale functions for $q=0$ and the classical ruin problem. Indeed setting $q=0$ and taking $a \uparrow \infty$ we have that $\mathbb{P}_{x}\left(\tau_{0}^{-}<\tau_{a}^{+}\right)=1-W(x) / W(\infty)$. Moreover, assuming that $\psi^{\prime}(0+)>0$, multiplying (5) through by $\theta$ and taking limits as $\theta \downarrow \infty$ to deduce that $W(\infty)^{-1}=\psi^{\prime}(0+)$, it follows that

$$
\mathbb{P}_{x}\left(\tau_{0}^{-}<\infty\right)=1-\psi^{\prime}(0+) W(x)
$$

It is in this context of ruin theory that scale functions make their earliest appearance in the works of Zolotarev (1964), Takács (1966) and then later either explicitly or implicitly in the work of Emery (1973) and Korolyuk (1974, 1975), Suprun (1976) and Rogers (1990). The real value of scale functions as a class with which one may express a whole range of fluctuation identities for spectrally negative Lévy processes became apparent in the work of Chaumont $(1994,1996)$ of Bertoin $(1996,1997)$ and an ensemble of subsequent articles; see for example Lambert (2000), Avram et al. (2004), Pistorius (2003, 2004, 2005, 2007), Kyprianou and Palmowski (2006), Doney and Kyprianou (2006) and Doney (1991, 2005a, 2007). Moreover with the advent of these new fluctuation identities and a better understanding of the analytical properties of the function $W^{(q)}$ came the possibility of revisiting and solving a number of classical and modern problems from applied probability, but now with the underlying source of randomness being a general spectrally negative Lévy processes. For example, in the theory of optimal stopping Avram et al. (2002, 2004); Alili and Kyprianou (2005) and Kyprianou (2006), in the theory of optimal control Avram et al. (2007) and Loeffen (2007), in the theory of queuing and storage models Dube et al. (2004) and Bekker et al. (2008), in the theory of branching processes Bingham (1976) and Lambert (2007), in the theory of insurance risk and ruin Chiu and Yin (2005), Klüppelberg et al. (2004), Klüppelberg and Kyprianou (2006), Doney and Kyprianou (2006), Kyprianou and Palmowski (2007), Renaud and Zhou (2007), Loeffen (2007) and Kyprianou et al. (2009), in the theory of credit risk Hilberink and Rogers (2002) and Kyprianou and Surya (2007) and in the theory of fragmentation Krell (2007). 
Although scale functions are now firmly embedded within the theory of spectrally negative Lévy processes and their applications, and although there is a reasonable understanding of how they behave analytically (see for example the summary in Kyprianou (2006)), one of their main failings from a practical point of view is that there are limited number of examples which are available 'off-the-shelf' for modeling purposes.

The original purpose of this paper was to introduce a previously unknown family of scale functions. However, in doing so, we came across a number of additional examples which were seemingly less well known in the literature or are themselves new and concurrent with this article. We shall therefore initially spend some time documenting known examples of scale functions. It seemed then sensible to also document known general analytical properties of scale functions and this we have also done in Section 3.

Following that we shall expose a new family of explicit examples of scale functions as well specifying in detail the associated Lévy processes. By 'explicit' we mean that, as in the below, known examples, we are able to give exact analytical expressions for the scale functions. By 'specifying in detail' we mean that we shall describe the Lévy triple $(a, \sigma, \Pi)$ of the associated spectrally negative Lévy process and in particular it will turn out that a density for the Lévy measure can be obtained and we are able to identify it as a known function. Moreover, it turns out that the associated Lévy processes discussed may also be described as the independent sums of other, well known Lévy processes.

\section{Known examples of scale functions}

At the time of writing, and to the best of the authors' knowledge, there are essentially six main examples of explicit classes; three of which deal with the case of a compound Poisson process with negative jumps and positive drift.

1. Brownian motion with drift. The associated Laplace exponent is written $\psi(\theta)=\sigma^{2} \theta^{2} / 2+\mu \theta$, where $\sigma>0$ and $\mu \in \mathbb{R}$ then, for $x \geq 0$,

$$
W^{(q)}(x)=\frac{2}{\sqrt{2 q \sigma^{2}+\mu}} e^{-\mu x / \sigma^{2}} \sinh \left(\frac{x}{\sigma^{2}} \sqrt{2 q \sigma^{2}+\mu}\right)
$$

for $q \geq 0$, where in the case that $q=\mu=0$ the above expression is to be taken in the limiting sense.

2. Spectrally negative stable processes with stability parameter $\beta \in(1,2)$. When $\psi(\theta)=\theta^{\beta}$ with $\beta \in(1,2)$, we have for $x \geq 0$

$$
W^{(q)}(x)=\beta x^{\beta-1} E_{\beta, 1}^{\prime}\left(q x^{\beta}\right)
$$

for $q \geq 0$ where $E_{\beta, 1}(z)=\sum_{k \geq 0} z^{k} / \Gamma(1+\beta k)$ is the Mittag-Leffler function.

There also exists an expression for the scale function of the aforementioned $\beta$-stable process but now with a strictly positive drift $c>0$ (but only for the case $q=0$ ) implicitly in the paper of Furrer (1998). Indeed, by considering the expression there for the ruin probability one may extract the following,

$$
W(x)=\frac{1}{c}\left(1-E_{\beta-1}\left(-c x^{\beta-1}\right)\right)
$$

for $x \geq 0$. Note that by taking $c \downarrow 0$ one obtains an expression which agrees with (8), namely $x^{\beta-1} / \Gamma(\beta)$. 
3. Generally speaking it is possible to deal with a strictly positive linear drift minus a compound Poisson process with positive jumps with a rational Laplace transform. The calculations are however rather extensive. See for example Mordecki and Lewis (2005) where the scale function can be extracted from their expression for the ruin probability. As a special case of the latter one may consider the case of exponentially distributed jumps. Denote the drift rate by $c$, the rate of arrival of jumps by $\lambda>0$ and the parameter associated with the exponentially distributed jumps by $\mu>0$; that is to say $\psi(\theta)=c \theta-\lambda\left(1-\mu(\mu+\theta)^{-1}\right)$. If one assumes that $c-\lambda / \mu>0$ so that $\psi^{\prime}(0+)>0$ then we have for the case $q=0$ and $x \geq 0$,

$$
W(x)=\frac{1}{c}\left(1+\frac{\lambda}{c \mu-\lambda}\left(1-e^{\left(\mu-c^{-1} \lambda\right) x}\right)\right) .
$$

The expression for the scale function in the case that $q>0$ with exponentially distributed jumps, and indeed for the slightly more general case where an independent Gaussian component is added in, can in principle be extracted from Kou and Wang (2003).

4. This example is taken from Asmussen (2000) and again comes from the case of a known ruin probability giving a scale function for the case $q=0$ and positive drift. Consider a spectrally negative compound Poisson process whose jumps are exactly of size $\alpha \in(0, \infty)$, with arrival rate $\lambda>0$ and with positive drift $c>0$ such that $c-\lambda \alpha>0$. In that case, $\psi(\theta)=c \theta-\lambda\left(1-e^{-\alpha \theta}\right)$ and for $x \geq 0$ we have

$$
W(x)=\frac{1}{c} \sum_{n=1}^{\lfloor x / \alpha\rfloor} e^{-\lambda(\alpha n-x) / c} \frac{1}{n !}\left(\frac{\lambda}{c}\right)^{n}(\alpha n-x)^{n}
$$

where $\lfloor x / \alpha\rfloor$ is the integer part of $x / \alpha$.

5. The next example comes from Abate and Whitt (1999) who themselves refer to older work of Boxma and Cohen (1998).

Consider a unit-rate linear drift minus a compound Poisson process of rate $\lambda>0$ with jumps whose distribution $F$ has Laplace transform given by

$$
\int_{0}^{\infty} e^{-\theta x} F(d x)=1-\frac{\theta}{(\mu+\sqrt{\theta})(1+\sqrt{\theta})}
$$

for $\Re(\theta) \geq 0$. The latter Laplace transform corresponds to a random variable whose mean is equal to $\mu^{-1}>0$ and whose tail distribution takes the form

$$
F(x, \infty)=(2 x+1) \eta(x)-2 \sqrt{\frac{x}{\pi}}, x \geq 0
$$

when $\mu=1$ and otherwise when $\mu \neq 1$

$$
F(x, \infty)=\left(\frac{1}{1-\mu}\right)\left(\eta(x)-\mu \eta\left(x \mu^{2}\right)\right), x \geq 0
$$

where $\eta(x)=e^{x} \operatorname{erfc}(\sqrt{x})$. It is assumed that the underlying Lévy process drifts to $\infty$. Since $\mathbb{E}\left(X_{1}\right)=1-\lambda / \mu$ the latter assumption is tantamount to $\lambda / \mu<1$.

Said another way, we are interested in a spectrally negative Lévy process with Laplace exponent given by

$$
\psi(\theta)=\theta-\frac{\lambda \theta}{(\mu+\sqrt{\theta})(1+\sqrt{\theta})} .
$$


For the scale function with $q=0$ it is known that for $x \geq 0$

$$
W(x)=\frac{1}{1-\lambda / \mu}\left(1-\frac{\lambda / \mu}{\nu_{1}-\nu_{2}}\left(\nu_{1} \eta\left(x \nu_{2}^{2}\right)-\nu_{2} \eta\left(x \nu_{1}^{2}\right)\right)\right)
$$

and

$$
\nu_{1,2}=\frac{1+\mu}{2} \pm \sqrt{\left(\frac{1+\mu}{2}\right)^{2}-\left(1-\frac{\lambda}{\mu}\right) \mu} .
$$

6. The final two examples, both scale functions only for the case $q=0$, appeared very recently in the theory of positive self-similar Markov processes, see Chaumont et al. (2007). See also Chaumont and Caballero (2006) for the origin of the underling Lévy processes. The first example is the scale function which belongs to a spectrally negative Lévy process with no Gaussian component, whose Lévy measure takes the form

$$
\Pi(d y)=\frac{e^{(\beta-1) y}}{\left(e^{y}-1\right)^{\beta+1}} d y, y<0
$$

where $\beta \in(1,2)$ and whose Laplace exponent takes the form

$$
\psi(\theta)=\frac{\Gamma(\theta-1+\beta)}{\Gamma(\theta-1) \Gamma(\beta)}
$$

for $\Re(\theta) \geq 0$. Note that $\psi^{\prime}(0+)<0$ and hence the process drifts to $-\infty$. In that case it was found that for $x \geq 0$

$$
W(x)=\left(1-e^{-x}\right)^{\beta-1} e^{x} .
$$

The second example is the scale function associated with the aforementioned Lévy process when conditioned to drift to $\infty$. It follows that there is still no Gaussian component and the Lévy measure takes the form

$$
\Pi(d y)=\frac{e^{\beta y}}{\left(e^{y}-1\right)^{\beta+1}} d y, y<0
$$

and the associated Laplace exponent is given by

$$
\psi(\theta)=\frac{\Gamma(\theta+\beta)}{\Gamma(\theta) \Gamma(\beta)}
$$

for $\Re(\theta) \geq 0$. The scale function is then given for $x \geq 0$ by

$$
W(x)=\left(1-e^{-x}\right)^{\beta-1} .
$$

\section{Known analytic properties of scale functions.}

Although at the time of writing further examples other than those above are lacking, there are a collection of general properties known for scale functions, mostly concerning their behaviour at 0 and $\infty$. For later reference in this text and to give credibility to some we review them briefly here. As usual, $(a, \sigma, \Pi)$ denotes the Lévy triple of a general spectrally negative Lévy process.

Smoothness The following facts are taken from Lambert (2000), Chan et al. (2009), Kyprianou et al. (2009) and Doney (2005b). It is known that if $X$ has paths of bounded variation then, for all $q \geq 0,\left.W^{(q)}\right|_{(0, \infty)} \in C^{1}(0, \infty)$ if and only if $\Pi$ has no atoms. In the case that $X$ has paths of unbounded variation, it is known that, for all $q \geq 0,\left.W^{(q)}\right|_{(0, \infty)} \in C^{1}(0, \infty)$. Moreover if $\sigma>0$ then $C^{1}(0, \infty)$ may be replaced by $C^{2}(0, \infty)$. It was also noted by Renming Song (see the remarks in Chan et al. (2009)) that if $\Pi(-\infty,-x)$ is completely monotone then $\left.W^{(q)}\right|_{(0, \infty)} \in C^{\infty}(0, \infty)$. 
Concavity and convexity It was shown in Loeffen (2007) that the latter assumption that $\Pi(-\infty,-x)$ is completely monotone also implies that $W^{(q)^{\prime}}(x)$ is convex for $q>0$. Note in particular, the latter implies that there exists an $a^{*} \geq 0$ such that $W^{(q)}$ is concave on $\left(0, a^{*}\right)$ and convex on $\left(a^{*}, \infty\right)$. In the case that $\psi^{\prime}(0+) \geq 0$ and $q=0$ the argument in Chan et al. (2009) shows that $a^{*}=\infty$ and $W$ is necessarily concave. In Kyprianou et al. (2009) it is shown that if $\Pi(-\infty,-x)$ has a density on $(0, \infty)$ which is non-increasing and log-convex then for each $q \geq 0$, the scale function $W^{(q)}(x)$ and its first derivative are convex beyond some finite value of $x$.

Continuity at the origin For all $q \geq 0$,

$$
W^{(q)}(0+)= \begin{cases}0 & \text { if } \sigma>0 \text { or } \int_{(-1,0)}(-x) \Pi(d x)=\infty \\ c^{-1} & \text { if } \sigma=0 \text { and } \int_{(-1,0)}(-x) \Pi(d x)<\infty\end{cases}
$$

where $\mathrm{c}=-a-\int_{(-1,0)} x \Pi(d x)$.

Derivative at the origin For all $q \geq 0$,

$$
W^{(q) \prime}(0+)= \begin{cases}2 / \sigma^{2} & \text { if } \sigma>0 \\ \infty & \text { if } \sigma=0 \text { and } \Pi(-\infty, 0)=\infty \\ (q+\Pi(-\infty, 0)) / \mathrm{c}^{2} & \text { if } \sigma=0 \text { and } \Pi(-\infty, 0)<\infty\end{cases}
$$

Behaviour at $\infty$ for $q=0$ As $x \uparrow \infty$ we have

$$
W(x) \sim \begin{cases}1 / \psi^{\prime}(0+) & \text { if } \psi^{\prime}(0+)>0 \\ e^{\Phi(0) x} / \psi^{\prime}(\Phi(0)) & \text { if } \psi^{\prime}(0+)<0\end{cases}
$$

When $\mathbb{E}\left(X_{1}\right)=0$ a number of different asymptotic behaviours may occur. For example, if $\phi(\theta):=\psi(\theta) / \theta$ satisfies $\phi^{\prime}(0+)<\infty$ then $W(x) \sim x / \phi^{\prime}(0+)$ as $x \uparrow \infty$.

Behaviour at $\infty$ for $q>0$ As $x \uparrow \infty$ we have

$$
W^{(q)}(x) \sim e^{\Phi(q) x} / \psi^{\prime}(\Phi(q))
$$

and thus there is asymptotic exponential growth.

Note that the last three properties can be extracted from the formula (6) and the the definition of $W^{(q)}$ through its Laplace transform (5). See Chapter 8 of Kyprianou (2006) for further details.

\section{Methodology for new examples}

For any given spectrally negative Lévy process, the scale functions are intimately connected to descending ladder height process and this forms the key to constructing new examples. Therefore we shall briefly review the connection between scale functions and the descending ladder height process before describing the common methodology that leads to the new examples of scale functions. For a more detailed account of this connection, the reader is referred to the books of Bertoin (1996), Kyprianou (2006) or Doney (2007).

It is straightforward to show that the process $X-\underline{X}:=\left\{X_{t}-\underline{X}_{t}: t \geq 0\right\}$, where $\underline{X}_{t}:=$ $\inf _{s \leq t} X_{s}$, is a strong Markov process with state space $[0, \infty)$. Following standard theory of Markov local times (cf. Chapter IV of Bertoin (1996)), it is possible to construct a local time at zero for $X-\underline{X}$ which we henceforth refer to as $L=\left\{L_{t}: t \geq 0\right\}$. Its inverse process, $L^{-1}:=\left\{L_{t}^{-1}: t \geq 0\right\}$ where $L_{t}^{-1}=\inf \left\{s>0: L_{s}>t\right\}$, is a (possibly killed) subordinator. 
Sampling $X$ at $L^{-1}$ we recover the points of minima of $X$. If we define $H_{t}=X_{L_{t}^{-1}}$ when $L_{t}^{-1}<\infty$ with $H_{t}=\infty$ otherwise, then it is known that the process $H=\left\{H_{t}: t \geq 0\right\}$ is a (possibly killed) subordinator. The latter is known as the descending ladder height process. Moreover, if $\Upsilon$ is the Lévy measure of $H$ then

$$
\Upsilon(x, \infty)=e^{\Phi(0) x} \int_{x}^{\infty} e^{-\Phi(0) u} \Pi(-\infty,-u) d u \text { for } x>0
$$

see for example Vigon (2002a). Further, the subordinator has a drift component if and only if $\sigma>0$ in which case the drift is necessarily equal to $\sigma^{2} / 2$. The killing rate of $H$ is given by the constant $\mathbb{E}\left(X_{1}\right) \vee 0$.

The starting point for the relationship between the descending ladder height process and scale functions is given by the Wiener-Hopf factorization. In 'Laplace form' for spectrally negative Lévy processes this takes the appearance

$$
\psi(\theta)=(\theta-\Phi(0)) \phi(\theta)
$$

where

$$
\phi(\theta)=-\log \mathbb{E}\left(e^{-\theta H_{1}}\right), \quad \psi(\theta)=\log \mathbb{E}\left(e^{\theta X_{1}}\right),
$$

and $\Re(\theta) \geq 0$. With this form of the Wiener-Hopf factorization in mind, we appeal principally to two techniques.

1. We choose the process $H$, or equivalently $\phi(\theta)$, so that the Laplace inversion of (5) may be performed. In some cases, when the process does not drift to $-\infty$ (or equivalently $\left.\psi^{\prime}(0+) \geq 0\right)$, it can be worked to ones advantage that, after an integration by parts, one also has that

$$
\int_{0}^{\infty} e^{-\theta x} W(d x)=\frac{1}{\phi(\theta)}
$$

for $\Re(\theta)>0$.

2. We choose the process $H$ to be such that its semigroup $\mathbb{P}\left(H_{t} \in d x\right)$ is known in explicit form and then make use of the following identity (see for example Bertoin (1996) or Kyprianou (2006)),

$$
\int_{0}^{\infty} d t \cdot \mathbb{P}\left(H_{t} \in d x\right)=W(d x), x \geq 0
$$

whenever $X$ does not drift to $-\infty$.

Naturally, forcing a choice of descending Ladder height process, or equivalently $\phi$, requires one to know that a spectrally negative Lévy process, $X$, exists whose Laplace exponent respects the factorization (28). The next Theorem provides the necessary justification. Indeed, to some extent it shows how to construct a Lévy process with a given descending ladder height process as well as a prescribed ascending ladder height process. For spectrally negative Lévy processes, the ascending ladder height process is a (killed) linear unit drift and has only a single parameter, namely $\Phi(0)$, the killing rate. Hence the construction in the below theorem offers a parameter $\varphi$ which plays the role of $\Phi(0)$. Since both ascending and descending ladder height process cannot both be killed one also sees the parameter restriction

$$
\varphi \kappa=0
$$

where $\kappa$ is the killing rate of the descending ladder height process. 
Theorem 1. Suppose that $H$ is a subordinator, killed at rate $\kappa \geq 0$, with Lévy measure which is absolutely continuous with non-increasing density and drift $\zeta$. Suppose further that $\varphi \geq 0$ is given such that $\varphi \kappa=0$. Then there exists a spectrally negative Lévy process $X$, henceforth referred to as the 'parent process', such that for all $x \geq 0, \mathbb{P}\left(\tau_{x}^{+}<\infty\right)=e^{-\varphi x}$ and whose descending ladder height process is precisely the process $H$. The Lévy triple $(a, \sigma, \Pi)$ of the parent process is uniquely identified as follows. The Gaussian coefficient is given by $\sigma=\sqrt{2 \zeta}$. The Lévy measure is given by

$$
\Pi(-\infty,-x)=\varphi \Upsilon(x, \infty)+\frac{d \Upsilon(x)}{d x}
$$

Finally

$$
a=\int_{(-\infty,-1)} x \Pi(d x)-\kappa
$$

if $\varphi=0$ and otherwise when $\varphi>0$ we can establish the value of a from the equation

$$
a \varphi=\frac{1}{2} \sigma^{2} \varphi^{2}+\int_{(-\infty, 0)}\left(e^{\varphi x}-1-x \varphi \mathbf{1}_{\{x>-1\}}\right) \Pi(d x) .
$$

In all cases, the Laplace exponent of the parent process is also given by

$$
\psi(\theta)=(\theta-\varphi) \phi(\theta)
$$

for $\theta \geq 0$ where $\phi(\theta)=-\log \mathbb{E}\left(e^{-\theta H_{1}}\right)$.

Proof. The proof is reasonably self evident given the preceding account of the ladder height process. One needs only the additional information that if $X$ is any spectrally negative Lévy process with Lévy measure $\Pi$ then

$$
\mathbb{E}\left(X_{1}\right)=-a+\int_{(-\infty,-1)} x \Pi(d x) .
$$

Moreover when $\Phi(0)>0$ then necessarily the descending ladder height process has no killing and when the descending ladder height process is killed then $\Phi(0)=0$. Further, for all $x \geq 0$ $\mathbb{P}\left(\tau_{x}^{+}<\infty\right)=e^{-\Phi(0) x}$.

The idea of working 'backwards' through the Wiener-Hopf factorization as we have done above can also be found in Bertoin et al. (2004) and Vigon (2002b). Note that it is more practical to describe the parent process in terms of the triple $(\sigma, \Pi, \psi)$ than the triple $(a, \sigma, \Pi)$ and we shall frequently do this in the sequel. It is also worth making an observation for later reference concerning the path variation of the process $X$ for a given a descending ladder height process $H$.

Corollary 1. Given a killed subordinator $H$ satisfying the conditions of the previous Theorem,

(i) the parent process has paths of unbounded variation if and only if $\Upsilon(0, \infty)=\infty$ or $\zeta>0$,

(ii) if $\Upsilon(0, \infty)=\lambda<\infty$ then the parent process necessarily decomposes in the form

$$
X_{t}=(\kappa+\lambda-\zeta \varphi) t+\sqrt{2 \zeta} B_{t}-S_{t}
$$

where $B=\left\{B_{t}: t \geq 0\right\}$ is a Brownian motion, $S=\left\{S_{t}: t \geq 0\right\}$ is an independent driftless subordinator with Lévy measure $\nu$ satisfying $\nu(x, \infty)=\Pi(-\infty,-x)$. 
Proof. The path variation of $X$ follows directly from (33) and the fact that $\sigma=\sqrt{2 \zeta}$. Also using (33), the Laplace exponent of the decomposition (38) can be computed as follows with the help of an integration by parts;

$$
\begin{aligned}
& (\kappa+\lambda-\zeta \varphi) \theta+\zeta \theta^{2}-\varphi \theta \int_{0}^{\infty} e^{-\theta x} \Upsilon(x, \infty) d x-\theta \int_{0}^{\infty} e^{-\theta x} \frac{d \Upsilon}{d x}(x) d x \\
& \quad=(\kappa+\Upsilon(0, \infty)-\zeta \varphi) \theta+\zeta \theta^{2}-\varphi \int_{0}^{\infty}\left(1-e^{-\theta x}\right) \frac{d \Upsilon}{d x}(x) d x-\theta \int_{0}^{\infty} e^{-\theta x} \frac{d \Upsilon}{d x}(x) d x \\
& \quad=(\theta-\varphi)\left(\kappa+\theta \zeta+\int_{0}^{\infty}\left(1-e^{-\theta x}\right) \frac{d \Upsilon}{d x}(x) d x\right) .
\end{aligned}
$$

This agrees with the Laplace exponent $\psi(\theta)=(\theta-\varphi) \phi(\theta)$ of the parent process constructed in Theorem 1.

\section{The Gaussian tempered stable convolution class}

In this paper we introduce a new family of spectrally negative Lévy processes from which our new examples of scale functions will emerge. We call them Gaussian tempered stable convolution, GTSC for short. When there is no Gaussian part we call the distribution a tempered stable convolution and write TSC for short.

\subsection{The tempered stable ladder process}

The starting point for the construction is a tempered stable subordinator plus a linear drift, possibly killed, that will play the role of the descending ladder height process for the GTSC parent process. Some references for tempered stable distributions and tempered stable Lévy processes are Rosiński (2002), Cont and Tankov (2003), Schoutens (2003) and Steutel and van Harn (2004). Note, that several names and origins, and many different parameterisations are used for the tempered stable distributions and processes.

In our parametrization, the tempered stable subordinator involves three parameters, the stability parameter $\alpha<1$, the tempering parameter $\gamma \geq 0$, and the scaling parameter $c>0$. When $\alpha \leq 0$ it is required that $\gamma>0$. Furthermore we might add a linear drift with rate $\zeta \geq 0$ to the process, and possibly kill the process at rate $\kappa \geq 0$.

The Laplace exponent of the tempered stable subordinator, and henceforth the descending ladder height process is thus taken to be ${ }^{1}$

$$
\phi(\theta)=\kappa+\zeta \theta+c \Gamma(-\alpha)\left(\gamma^{\alpha}-(\gamma+\theta)^{\alpha}\right), \quad \Re(\theta)>-\gamma,
$$

and the associated Lévy measure is given by

$$
\Upsilon(d x)=c x^{-\alpha-1} e^{-\gamma x} d x \quad(x>0) .
$$

For $0 \leq \alpha<1$ the process has infinite activity. For $\alpha=0$ the expression (39) is to be understood in a limiting sense, i.e., $\phi(\theta)=\kappa+\zeta \theta-c \log (\gamma /(\gamma+\theta))$, and the process is simply a (killed) gamma subordinator (with drift). If $\alpha<0$ the process is a compound Poisson process with intensity parameter $c \Gamma(-\alpha) \gamma^{\alpha}$ and gamma distributed jumps with $-\alpha$ degrees of freedom and exponential parameter $\gamma>0$.

\footnotetext{
${ }^{1}$ Note that we use $\Gamma(z)$ as a meromorphic function with simple poles at the non-positive integers. By the functional equation of the gamma function we have $\Gamma(-\alpha)=-\alpha^{-1} \Gamma(1-\alpha)$, and $\Gamma(-\alpha)<0$ for $\alpha \in(0,1)$.
} 


\subsection{The associated parent process}

We may now invoke Theorem 1 to construct the associated GTSC process. This introduces another parameter $\varphi \geq 0$. To be able to apply the theorem, we need a decreasing Lévy density, and thus we have to restrict the scaling parameter to $-1 \leq \alpha<1$. The theorem tells us that the Laplace exponent of the parent process is

$$
\psi(\theta)=(\kappa-\varphi \zeta) \theta+\zeta \theta^{2}+c(\theta-\varphi) \Gamma(-\alpha)\left(\gamma^{\alpha}-(\gamma+\theta)^{\alpha}\right)
$$

for $\Re(\theta)>\gamma$, and obviously for $\alpha=0$ we understand the above expression in the limiting sense so that $\psi(\theta)=(\kappa-\zeta \varphi) \theta+\zeta \theta^{2}-c(\theta-\varphi) \log (\gamma / \gamma+\theta)$. It is important to recall here and throughout the remainder of the paper that $\kappa \varphi=0$. The corresponding Lévy measure given by

$$
\Pi(d x)=c \frac{(\varphi+\gamma)}{(-x)^{\alpha+1}} e^{\gamma x} d x+c \frac{(\alpha+1)}{(-x)^{\alpha+2}} e^{\gamma x} d x
$$

for $x<0$. This indicates that the jump part is the result of the independent sum of two spectrally negative tempered stable processes with stability parameters $\alpha$ and $\alpha+1$. We also note from Theorem 1 that $\sigma=\sqrt{2 \zeta}$, indicating the presence of a Gaussian component. This motivates the choice of terminology Gaussian Tempered Stable Convolution.

If $0<\alpha<1$ the jump component is the sum of an infinite activity negative tempered stable subordinator and an independent spectrally negative tempered stable process with infinite variation. If $\alpha=0$ the jump component is the sum of a spectrally negative infinite variation tempered stable process with stability parameter 1 and exponential parameter $\gamma$ and the negative of a gamma subordinator with exponential parameter $\gamma$. If $-1 \leq \alpha<0$ the jump part of the parent process is the independent sum tempered stable subordinator with stability parameter $1+\alpha$ and exponential parameter $\gamma$, and an independent negative compound Poisson subordinator with jumps from a gamma distribution with $-\alpha$ degrees of freedom and exponential parameter $\gamma$. In the extreme case $\alpha=-1$, the parent process has negative jumps which are compound Poisson and exponentially distributed with parameter $\gamma$.

\section{Evaluating GTSC scale and $q$-scale functions}

Let us momentarily introduce some notation which will be repeatedly used throughout the remainder of the paper. We define the generalized Mittag-Leffler function with indices $\alpha>0$ and $\beta>0$ by

$$
E_{\alpha, \beta}(z)=\sum_{n \geq 0} \frac{z^{n}}{\Gamma(\alpha n+\beta)}
$$

Note that the latter is an entire on $z \in \mathbb{C}$. For $k=0,1,2, \ldots$ we define $E_{\alpha, \beta}^{(k)}(z)$ to be the $k$-th derivative of $E_{\alpha, \beta}(z)$ (with the obvious meaning when $k=0$ ).

\subsection{The case with rational nonzero stability parameter}

Theorem 2. Suppose $\alpha=m / n$ with $m \in \mathbb{Z}, n \in \mathbb{Z}, 0<|m|<n$, and $\operatorname{gcd}(|m|, n)=1$. Let us consider the GTSC process with Laplace exponent

$$
\psi(\theta)=(\theta-\varphi)\left[\kappa+\zeta \theta+c \Gamma(-\alpha)\left(\gamma^{\alpha}-(\gamma+\theta)^{\alpha}\right)\right] .
$$

Let $m_{+}=\max (m, 0)$ and $m_{-}=\max (-m, 0)$. Then the polynomial

$$
\left.f_{q}(z)=\left(z^{n}-\gamma-\varphi\right)\left[\left(\kappa+\zeta \gamma+c \Gamma(-\alpha) \gamma^{\alpha}\right) z^{m_{-}}+\zeta z^{n+m_{-}}-c \Gamma(-\alpha) z^{m_{+}}\right)\right]-q z^{m_{-}}
$$


has at least one real root. Let $\ell$ denote the number of distinct roots, $r_{1}, \ldots, r_{\ell}$ the roots, arranged in some (arbitrary) order, and $\mu_{1}, \ldots, \mu_{\ell}$ the corresponding multiplicities. Let $A_{k j}$ for $k=$ $1, \ldots, \ell, j=0, \ldots, \mu_{k}-1$ denote the coefficients in the partial fraction decomposition

$$
\frac{z^{m_{-}}}{f_{q}(z)}=\sum_{k=1}^{\ell} \sum_{j=0}^{\mu_{k}-1} \frac{A_{k j}}{\left(z-r_{k}\right)^{j+1}}
$$

Then we have

$$
\Phi(q)=r_{*}^{n}-\gamma
$$

where $r_{*}$ denotes the largest real root, and

$$
W^{(q)}(x)=e^{-\gamma x} \sum_{k=1}^{\ell} \sum_{j=0}^{\mu_{k}-1} A_{k j} \frac{1}{j !} x^{(j+1) / n-1} E_{\frac{1}{n}, \frac{1}{n}}^{(j)}\left(r_{k} x^{\frac{1}{n}}\right) .
$$

Proof. We have

$$
f_{q}(z)=z^{m_{-}}\left(\psi\left(z^{n}-\gamma\right)-q\right)
$$

for $z \in \mathbb{Z}$ with $|\arg (z)|<\pi / n$, and conversely,

$$
\frac{1}{\psi(\theta)-q}=\frac{(\theta+\gamma)^{\frac{m_{-}}{n}}}{f_{q}\left((\theta+\gamma)^{\frac{1}{n}}\right)}
$$

for $\theta \in \mathbb{C} \backslash(-\infty,-\gamma]$ such that the denominator on the left hand side does not vanish. We know from the theory of scale functions that $\theta=\Phi(q)$ is a real root of $\psi(\theta)-q=0$, and there is no larger real root of that equation. Thus $z=(\Phi(q)+\gamma)^{\frac{1}{n}}$ is a real root of $f_{q}(z)=0$ and there is no larger real root of that equation. This shows (47).

The left hand side of (50) is the Laplace transform of $W^{(q)}(x)$, and we know it is an analytic function for $\Re(\theta)>\Phi(q)$. Using the partial fraction decomposition we get

$$
\int_{0}^{\infty} e^{-\theta x} W^{(q)}(x) d x=\sum_{k=1}^{\ell} \sum_{j=0}^{\mu_{k}-1} \frac{A_{k j}}{\left((\gamma+\theta)^{\frac{1}{n}}-r_{k}\right)^{j+1}},
$$

and this equation is valid for any $q \geq 0$ and $\Re(\theta)>\Phi(q)$. We recognize the Laplace transform of exponentially tilted derivatives of Mittag-Leffler functions on the right hand side, see for example (Jacob, 2005, Prop.7.1.9, p.359), and obtain (48).

Generally speaking this is rather an awkward formula to work with analytically. The main strength of the expression lies with it being a simple matter to program into a package such as MATLAB or Mathematica. It is instructive to revisit the (known) analytical properties of $W(x)$ and $W^{(q)}(x)$ listed in section 3 and check them directly for our explicit expression as this will yield in most cases more detail.

Smoothness: Since the Mittag-Leffler functions are entire functions, we see that $W^{(q)}(x)$, which is a priori defined for $x \in(0, \infty)$, admits an analytic continuation to $x \in \mathbb{C} \backslash(-\infty, 0]$, and thus $W^{(q)}$ is $C^{\infty}$ on $(0, \infty)$.

Behaviour at zero: We obtain the behaviour at zero from the power series expansion of the derivatives of the Mittag-Leffler function. Exploiting algebraic relations of the $r_{k}$ and $A_{k j}$ several terms cancel and we obtain for $\zeta>0$

$$
W^{(q)}(x) \sim \frac{x}{\zeta}, \quad W^{(q) \prime}(x) \sim \frac{1}{\zeta} \quad(x \rightarrow 0) .
$$


A similar argument applies for $\zeta=0$. If $0<\alpha<1$ we get

$$
W^{(q)}(x) \sim-\frac{x^{\alpha}}{c \Gamma(-\alpha) \Gamma(1+\alpha)}, \quad W^{(q) \prime}(x) \sim-\frac{x^{\alpha-1}}{c \Gamma(-\alpha) \Gamma(\alpha)}, \quad(x \rightarrow 0) .
$$

In the case that $-1<\alpha<0$ and $\zeta=0$. we get again by cancellation of terms and limiting behaviour at zero of Mittag-Leffler functions

$$
W^{(q)}(x) \sim \frac{1}{\kappa+c \gamma^{\alpha} \Gamma(-\alpha)}, \quad W^{(q) \prime}(x) \sim \frac{c x^{-\alpha-1}}{\left(\kappa+c \gamma^{\alpha} \Gamma(-\alpha)\right)^{2}}, \quad(x \rightarrow 0) .
$$

Note, another way to establish these last two results is by Karamata's Tauberian Theorem and the Monotone Density Theorem respectively from the asymptotics of $\psi(\theta)$ as $\theta \rightarrow \infty$. This approach shows, that the asymptotics above also hold for irrational $\alpha \in(-1,1)$.

Behaviour at infinity: For notational simplicity we assume in this paragraph (without loss of generality), that $r_{*}=r_{1}$. The behaviour at infinity can then be obtained from the asymptotics of the Mittag-Leffler functions at infinity. The dominating contribution comes from the term with $r_{1}$. It is useful to recall that $f_{q}(z)=\psi\left(z^{n}-\gamma\right)-q$ and thus $f_{q}^{\prime}(z)=n z^{n-1} \psi^{\prime}\left(z^{n}-\gamma\right)$. Let us consider first $q=0$. Note, that we have $\psi^{\prime}(0+)=\kappa-\varphi\left(\zeta+c \gamma^{\alpha-1} \Gamma(1-\alpha)\right)$. Suppose $\psi^{\prime}(0+)>0$, which implies that $\varphi=0$. Then $r_{1}=\gamma^{\frac{1}{n}}$ is a single root of $f_{0}(z)$. After some elementary simplifications we obtain

$$
W(x) \sim \frac{1}{\kappa} \quad(x \rightarrow \infty)
$$

in agreement with the theory. Suppose now $\psi^{\prime}(0+)<0$. Then $r_{1}=(\varphi+\gamma)^{\frac{1}{n}}$ is a simple root of $f_{0}(z)$. As above, using the asymptotics of the Mittag-Leffler functions at infinity, we see that the term with $r_{1}$ dominates all other terms. After elementary simplifications we obtain

$$
W(x) \sim \frac{e^{\varphi x}}{\kappa+\zeta \varphi+c \Gamma(-\alpha)\left(\gamma^{\alpha}-(\gamma+\varphi)^{\alpha}\right)} \quad(x \rightarrow \infty) .
$$

Consider the third case, $\psi^{\prime}(0+)=0$. This can only happen when $\kappa=0$ and $\varphi=0$. Then $r_{1}=\gamma^{\frac{1}{n}}$ is a double root of $f_{0}(z)$. The asymptotics show, that the contribution from $r_{1}$ dominates and we obtain

$$
W(x) \sim \frac{x}{\zeta+c \gamma^{\alpha-1} \Gamma(1-\alpha)} \quad(x \rightarrow \infty) .
$$

Finally in the case $q>0$, little more can be said than (26).

\subsection{The case with general nonzero stability parameter $\alpha \in(-1,1)$}

When we take $q=0$ and $\zeta=0$ and $\alpha \in(-1,1) \backslash\{0\}$ (without the restriction of begin a rational number) one may obtain much cleaner expressions for the scale function than the formulation in Theorem 2.

Theorem 3. Suppose $\alpha \in(-1,1) \backslash\{0\}$ and consider a TSC process (without Gaussian component) and Laplace exponent

$$
\psi(\theta)=(\theta-\varphi)\left[\kappa+c \Gamma(-\alpha)\left(\gamma^{\alpha}-(\gamma+\theta)^{\alpha}\right)\right] .
$$

If $0<\alpha<1$ then

$$
W(x)=-\frac{e^{\varphi x}}{c \Gamma(-\alpha)} \int_{0}^{x} e^{-(\gamma+\varphi) y} y^{\alpha-1} E_{\alpha, \alpha}\left(\frac{\kappa+c \Gamma(-\alpha) \gamma^{\alpha} y^{\alpha}}{c \Gamma(-\alpha)}\right) d y
$$


If $-1<\alpha<0$, then

$$
\begin{aligned}
W(x)= & \frac{e^{\varphi x}}{\kappa+c \Gamma(-\alpha) \gamma^{\alpha}} \\
& +\frac{c \Gamma(-\alpha) e^{\varphi x}}{\left(\kappa+c \Gamma(-\alpha) \gamma^{\alpha}\right)^{2}} \int_{0}^{x} e^{-(\gamma+\varphi) y} y^{-\alpha-1} E_{-\alpha,-\alpha}\left(\frac{c \Gamma(-\alpha) y^{-\alpha}}{\kappa+c \Gamma(-\alpha) \gamma^{\alpha}}\right) d y .
\end{aligned}
$$

Proof. This follows from the known Laplace transforms

$$
\int_{0}^{\infty} e^{-\theta x} x^{\alpha-1} E_{\alpha, \alpha}\left(\lambda x^{\alpha}\right) d x=\frac{1}{\theta^{\alpha}-\lambda}
$$

and

$$
\int_{0}^{\infty} e^{-\theta x} \lambda^{-1} x^{-\alpha-1} E_{-\alpha,-\alpha}\left(\lambda^{-1} x^{-\alpha}\right) d x=\frac{\lambda}{\lambda-\theta^{\alpha}}-1,
$$

valid for $\alpha>0$ resp. $\alpha<0$ together with the well-known rules for Laplace transforms concerning, primitives and tilting.

With these closed-form expressions, the explicit asymptotics discussed after Theorem 2 are immediate by inspection. Note in the particular case that $\kappa=-c \Gamma(-\alpha) \gamma^{\alpha}$ the expression for the scale function in the case $0<\alpha<1$ reduces simply to

$$
W(x)=\frac{1}{\kappa} \int_{0}^{x} \frac{\gamma^{\alpha}}{\Gamma(\alpha)} y^{\alpha-1} e^{-\gamma y} d y .
$$

Note that although the Laplace inversions involved in the proof of Theorem 3 are straightforward, it is not clear that the resulting expressions for $W$ are scale functions without the presence of Theorem 1 .

\subsection{The case with $\alpha=\frac{1}{2}$ : inverse Gaussian descending ladder height process}

We will assume now that $\zeta=0, \varphi=0, \kappa=0$. Substituting $\alpha \mapsto 1 / 2, c \mapsto \delta / \sqrt{2 \pi}, \gamma \mapsto \gamma^{2} / 2$ reveals that the tempered stable subordinator we have been working with is in fact the familiar inverse Gaussian $I G(\delta, \gamma)$-subordinator when $\alpha=1 / 2$. It turns out for this case that we also get some cleaner expressions than the one given in Theorem 2 .

When the descending ladder height process is the $I G(\delta, \gamma)$ subordinator, the jump part of the parent process is a superposition of a negative IG subordinator and a spectrally negative tempered stable process with stability parameter $3 / 2$.

Following the general approach in Theorem 2 we relate $\psi(\theta)$ to a polynomial, which, in the IG parametrization of this section, is $f_{q}(z)=\psi\left(z^{2}-\gamma^{2} / 2\right)-q$. The first simplification is, that $f_{q}(z)$ is a polynomial of degree 4 if $\zeta>0$, and degree 3 , if $\zeta=0$. Thus completely explicit, elementary (and somewhat lengthy) expressions of its roots can be given in terms of radicals by the formulæ of Ferrari resp. Cardano. The second simplification concerns the Mittag-Leffler functions. As $\alpha=1 / 2$ the Mittag-Leffler function simplifies to an expression involving the more familiar (complementary) error function.

We find that $f_{0}(z)$ has a positive double a negative single root, and after some elementary simplifications,

$$
W(x)=\frac{1}{2 \delta \gamma}\left[\left(1+\gamma^{2} x\right) \operatorname{erfc}(-\gamma \sqrt{x / 2})+\gamma \sqrt{2 x / \pi} e^{-\frac{1}{2} \gamma^{2} x}-1\right] .
$$


Actually, in this special case the result can be verified faster by calculating the Laplace transform of (64). As a side remark, we note, that $W^{\prime \prime}(x)=-x^{-3 / 2} e^{-\frac{1}{2} \gamma^{2} x} /(2 \sqrt{2 \pi} \delta)$ and thus we see, that $W$ is indeed concave, as we already know from the theory. Let us now consider $q>0$ and put

$$
q_{0}=\frac{16}{27} \delta \gamma^{3} .
$$

If $0<q<q_{0}$ then we have three simple real roots $r_{1}, r_{2}, r_{3}$, that can be obtained by Cardano's formula ${ }^{2}$, and

$$
W^{(q)}(x)=e^{-\frac{1}{2} \gamma^{2} x}\left[\frac{r_{1} e^{r_{1}^{2} x}}{f_{q}^{\prime}\left(r_{1}\right)} \operatorname{erfc}\left(-r_{1} \sqrt{x}\right)+\frac{r_{2} e^{r_{2}^{2} x}}{f_{q}^{\prime}\left(r_{2}\right)} \operatorname{erfc}\left(-r_{2} \sqrt{x}\right)+\frac{r_{3} e^{r_{3}^{2} x}}{f_{q}^{\prime}\left(r_{3}\right)} \operatorname{erfc}\left(-r_{3} \sqrt{x}\right)\right] .
$$

If $q>q_{0}$ we have a simple real root $r_{1}$ and two complex conjugate simple roots $r_{2}$ and $r_{3}$. The same formulas hold as in the previous case. Alternatively, we could in the complex case write

$$
W^{(q)}(x)=e^{-\frac{1}{2} \gamma^{2} x}\left[\frac{r_{1} e^{r_{1}^{2} x}}{f_{q}^{\prime}\left(r_{1}\right)} \operatorname{erfc}\left(-r_{1} \sqrt{x}\right)+2 \Re\left\{\frac{r_{2} e^{r_{2}^{2} x}}{f_{q}^{\prime}\left(r_{2}\right)} \operatorname{erfc}\left(-r_{2} \sqrt{x}\right)\right\}\right] .
$$

If $q=q_{0}$ then we have a simple positive and a double negative root, and after some easy simplifications,

$$
\begin{array}{r}
W^{\left(q_{0}\right)}(x)=\frac{1}{36 \delta \gamma}\left[6 \gamma \sqrt{\frac{2 x}{\pi}} e^{-\frac{1}{2} \gamma^{2} x}+15 e^{\frac{8}{9} \gamma^{2} x} \operatorname{erfc}\left(-\frac{5 \gamma}{3} \sqrt{\frac{x}{2}}\right)\right. \\
\left.-e^{-\frac{4}{9} \gamma^{2} x}\left(15+2 \gamma^{2} x\right) \operatorname{erfc}\left(\frac{\gamma}{3} \sqrt{\frac{x}{2}}\right)\right] .
\end{array}
$$

\subsection{The stable case}

Because of their definition via their Laplace transform, it is immediate that scale functions are continuous in the parameters of the parent process. Taking $\gamma \downarrow 0$ in the Laplace exponent of the tempered stable ladder height process shows us that the parent process is an $\alpha+1$ stable process when $\kappa=\varphi=\zeta=0$ and $c=-1 / \Gamma(-\alpha)$. Taking limits as $\gamma \downarrow 0$ in the expression for the scale function in the first part of Theorem 3 gives us the known result $W(x)=x^{\alpha} / \Gamma(\alpha+1)$.

\subsection{The case $\alpha=0$ : gamma descending ladder height process}

When $\alpha=0$ we can only treat the special case with $q=0, \kappa=0, \zeta=0$ and $\varphi=0$.

\section{Theorem 4.}

$$
W(x)=\int_{0}^{\infty} P(c t, \gamma x) d t,
$$

where $P(a, x)$ is the regularized lower incomplete gamma function.

\footnotetext{
${ }^{2}$ Explicitly$$
r_{1}=\frac{\gamma}{3 \sqrt{2}}+\frac{2 \sqrt{2} \delta \gamma^{2}}{3 \sqrt[3]{-8 \delta^{3} \gamma^{3}+27 \delta^{2} q+3 \sqrt{3} \sqrt{27 \delta^{4} q^{2}-16 \delta^{5} \gamma^{3} q}}}+\frac{\sqrt[3]{-8 \delta^{3} \gamma^{3}+27 \delta^{2} q+3 \sqrt{3} \sqrt{27 \delta^{4} q^{2}-16 \delta^{5} \gamma^{3} q}}}{3 \sqrt{2} \delta}
$$$$
r_{2}=\frac{\gamma}{3 \sqrt{2}}-\frac{\sqrt{2}(1+i \sqrt{3}) \delta \gamma^{2}}{3 \sqrt[3]{-8 \delta^{3} \gamma^{3}+27 \delta^{2} q+3 \sqrt{3} \sqrt{27 \delta^{4} q^{2}-16 \delta^{5} \gamma^{3} q}}}-\frac{(1-i \sqrt{3}) \sqrt[3]{-8 \delta^{3} \gamma^{3}+27 \delta^{2} q+3 \sqrt{3} \sqrt{27 \delta^{4} q^{2}-16 \delta^{5} \gamma^{3} q}}}{6 \sqrt{2} \delta}
$$$$
r_{3}=\frac{\gamma}{3 \sqrt{2}}-\frac{\sqrt{2}(1-i \sqrt{3}) \delta \gamma^{2}}{3 \sqrt[3]{-8 \delta^{3} \gamma^{3}+27 \delta^{2} q+3 \sqrt{3} \sqrt{27 \delta^{4} q^{2}-16 \delta^{5} \gamma^{3} q}}}-\frac{(1+i \sqrt{3}) \sqrt[3]{-8 \delta^{3} \gamma^{3}+27 \delta^{2} q+3 \sqrt{3} \sqrt{27 \delta^{4} q^{2}-16 \delta^{5} \gamma^{3} q}}}{6 \sqrt{2} \delta} .
$$

If $q=\delta \gamma^{3} / 2$ those formulae simplify, as one root is then zero.
} 
Proof. We may appeal to the second method in (31) using resolvents to note that the scale function density satisfies

$$
W^{\prime}(x)=\frac{1}{c} x^{-1} e^{-\gamma x} \varphi(-\log (\gamma x))
$$

where

$$
\varphi(\theta)=\int_{0}^{\infty} \frac{e^{-\theta x}}{\Gamma(x)} d x,
$$

which is the Laplace transform of the reciprocal gamma function. ${ }^{3}$ As $X$ has infinite variation, we have $W(0+)=0$. Integrating (70) yields the result.

\subsection{Remarks on the cases $\alpha=-1 / 2$ and $\alpha=-1$}

The case $\alpha=-1 / 2$ is again covered in Theorem 2 above, but several simplifications occur. The jump part of the ladder process is in this case a compound Poisson process with jumps from the $\chi_{1}$-distribution. Again, we can avoid Mittag-Leffler functions and use the complimentary error function instead. With regard to the roots of the polynomial equation we may say the following. If $\zeta>0$ the polynomial $f_{q}(z)$ has degree 5 and its roots typically cannot be expressed in terms of radicals. If $\zeta=0$ the polynomial is of degree 3 and Cardano's formula can be used. If in addition $\kappa=0$ and $\varphi=0$ then we have $r_{1}=\sqrt{\gamma}$ a double and $r_{2}=-\sqrt{\gamma}$ a single root. We can proceed as is Section 6.3 to obtain some simple closed form expressions for $W(x)$ and $W^{(q)}(x)$.

When $\alpha=-1$ the jump part of the parent process is a compound Poisson process with negative exponential jumps, and the ladder process is a compound Poisson process with exponential jumps. The Laplace transform of the scale functions are rational, and the scale functions can be obtained directly from the partial fraction decomposition. The results are implicitly contained in Mordecki and Lewis (2005) and Kou and Wang (2003) and we exclude the calculations here.

\section{Numerical illustrations}

Figure 1 contains graphs of the GTSC scale function $W(x)$ for stability parameters $\alpha=1 / 4$, $1 / 3,1 / 2,2 / 3,3 / 4$ in six cases. Figure 2 contains graphs of the GTSC $q$-scale functions $W^{(q)}(x)$ with $q=1$, but otherwise for the same parameters. Below we introduce six cases considered in terms of the classification of the parent process.

- Case A, $\kappa=0, \varphi=0, \zeta=0, c=1, \gamma=1$ : The parent process is oscillating, has no diffusion part and infinite variation jumps. The ladder process is an infinite activity pure jump subordinator, has no linear drift, and is not killed.

- Case B, $\kappa=1, \varphi=0, \zeta=0, c=1, \gamma=1$ : The parent process drifts to $+\infty$, has no diffusion part and infinite variation jumps. The ladder process is an infinite activity pure jump subordinator killed at rate $\kappa=1$.

- Case C, $\kappa=0, \varphi=1, \zeta=0, c=1, \gamma=1$ : The parent process drifts to $-\infty$, has no diffusion part and infinite variation jumps. The ladder process is an infinite activity pure jump subordinator.

- Case D, $\kappa=0, \varphi=0, \zeta=1, c=1, \gamma=1$ : The parent process is oscillating, has no linear drift, but a diffusion part and infinite variation jumps. The ladder process is an infinite

3 The value at $\theta=0$ is called the Fransén-Robertson constant and denoted by $\varphi(0)=F$. A lot of analytical and numerical material on $\varphi(\theta)$ can be found in Fransén and Wrigge (1984). That paper contains also several references to related work going back to Paley, Wiener, Hardy and Ramanujan. Note, that the reciprocal gamma distribution in Fransén and Wrigge (1984) is not to be confused with the inverse gamma distribution, which is often called reciprocal gamma distribution as well. 
activity pure jump subordinator plus a linear drift.

- Case E, $\kappa=1, \varphi=0, \zeta=1, c=1, \gamma=1$ : The parent process drifts to $+\infty$, has a Gaussian component and infinite variation jumps. The ladder process is an infinite activity subordinator plus linear drift, killed at unit rate.

- Case F, $\kappa=0, \varphi=1, \zeta=1, c=1, \gamma=1$ : The parent process drifts to $-\infty$, has a diffusion part and infinite variation jumps. The ladder process is an infinite activity pure jump subordinator plus a linear drift.

Now let us discuss the graphs in view of the theoretical properties listed in Section 3.

Smoothness: All graphs look smooth, as the theory predicts.

Concavity and convexity: For $q=0$ we know in cases A, B, D and E the graph is predicted to be concave. In cases $C$ and $F$ the graph is predicted to be convex-concave. Indeed this is the appearance in Figure 1.

For $q=1$ we know, that all graphs are concave-convex. We observe this behaviour in Figure 2 . In some cases, it is necessary to inspect the shape of the graph more closely in the neighbourhood of the origin in order to see concavity.

Behaviour at zero: For all cases we observe $W(0)=0$ in Figure 1 and $W^{(q)}(0)=0$ in Figure 2, in agreement with formula (23).

For the cases A-C (no diffusion part) we observe $W^{\prime}(0)=+\infty$, for the cases D-E (nonzero diffusion part) we observe $W^{\prime}(0)=1$ in Figure 1 . We observe the same behaviour, $W^{(q) \prime}(0)=\infty$ resp. $W^{(q) \prime}(0)=1$, in Figure 2. This is in agreement with formula (24).

Behaviour at infinity: Firstly let us consider the case $q=0$. In cases $\mathrm{A}$ and $\mathrm{D}$ we have $\psi^{\prime}(0+)=0$ and thus, according to (57), asymptotic linear growth as $x \rightarrow \infty$. In cases B and $\mathrm{E}$ we have $\kappa>0$ and hence $\psi^{\prime}(0+)>0$ so that $W(x) \rightarrow 1 / \kappa$ as $x \rightarrow \infty$. In cases $\mathrm{C}$ and $\mathrm{F}$ we have $\varphi>0$ and hence $\psi^{\prime}(0)<0$ and so there is exponential growth of $W(x)$ as $x \rightarrow \infty$ according to the second case in formula (25). This corresponds to what is observed in the graphs in Figure 1. (The behaviour for $x \rightarrow \infty$ becomes more prominent when plotting $0<x<20$.)

For the case $q>0$, all graphs in Figure 2 appear exhibit exponential growth as $x \rightarrow \infty$, in agreement with formula (26).

\section{Additional Remarks}

\subsection{Extensions and ramifications}

Our methods apply also to the integrated q-scale functions, see (Kyprianou, 2006, Theorem 8.1, p.214)

$$
Z^{(q)}(x)=1+q \int_{0}^{x} W^{(q)}(y) d y,
$$

for the Gaussian tempered stable convolution class with rational stability parameter.

Also we could treat more complicated Gaussian tempered stable convolutions with

$$
\phi(\theta)=\kappa+\zeta \theta+\sum_{i=1}^{n} c_{i} \Gamma\left(-\alpha_{i}\right)\left(\gamma_{i}^{\alpha_{i}}-\left(\gamma_{i}+\theta\right)^{\alpha_{i}}\right)
$$

with $\alpha_{1}, \ldots, \alpha_{n}$ rational numbers in $[-1,1)$. 

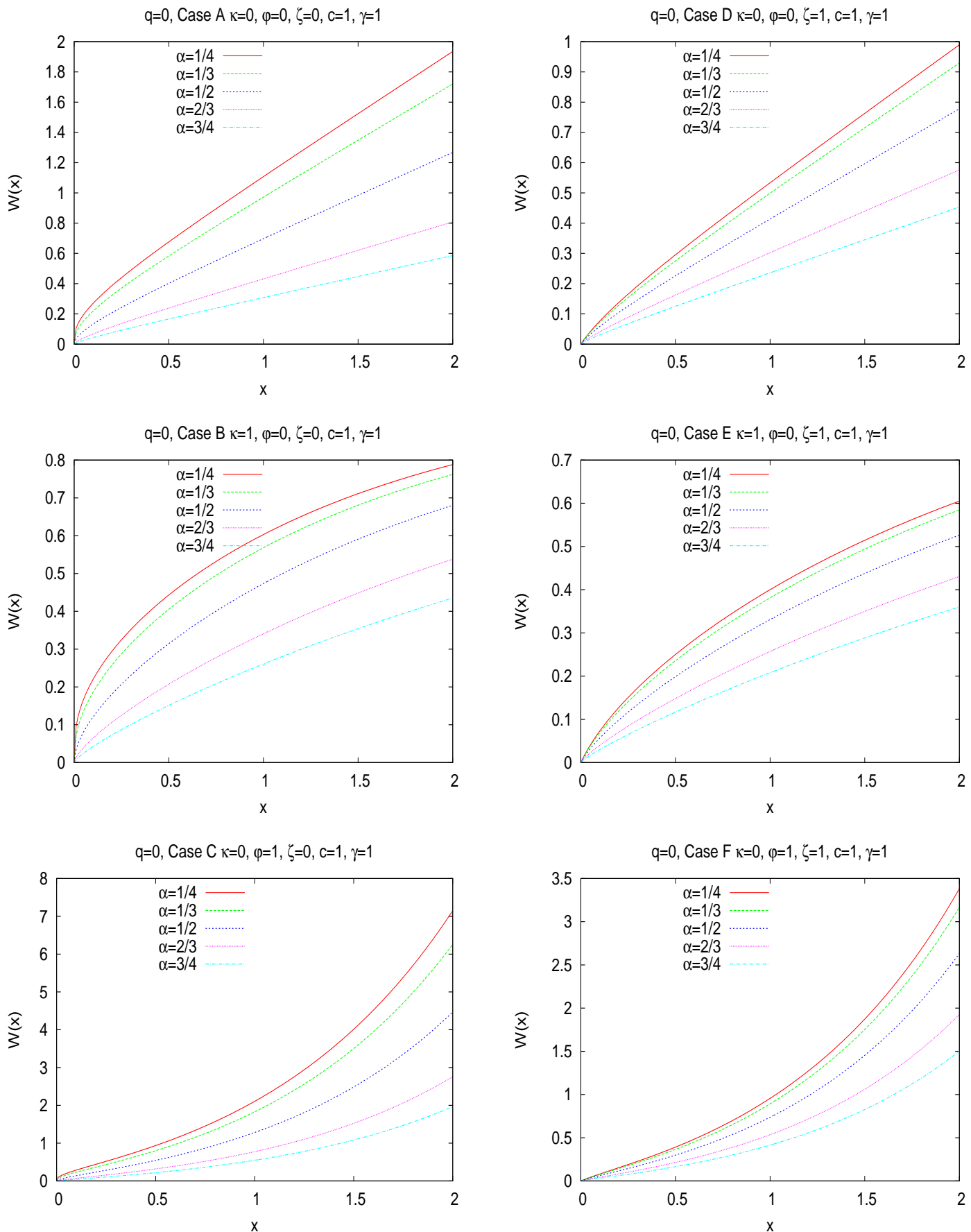

Figure 1: Scale functions $W(x)$ for the GTSC class with $0<\alpha<1$ 

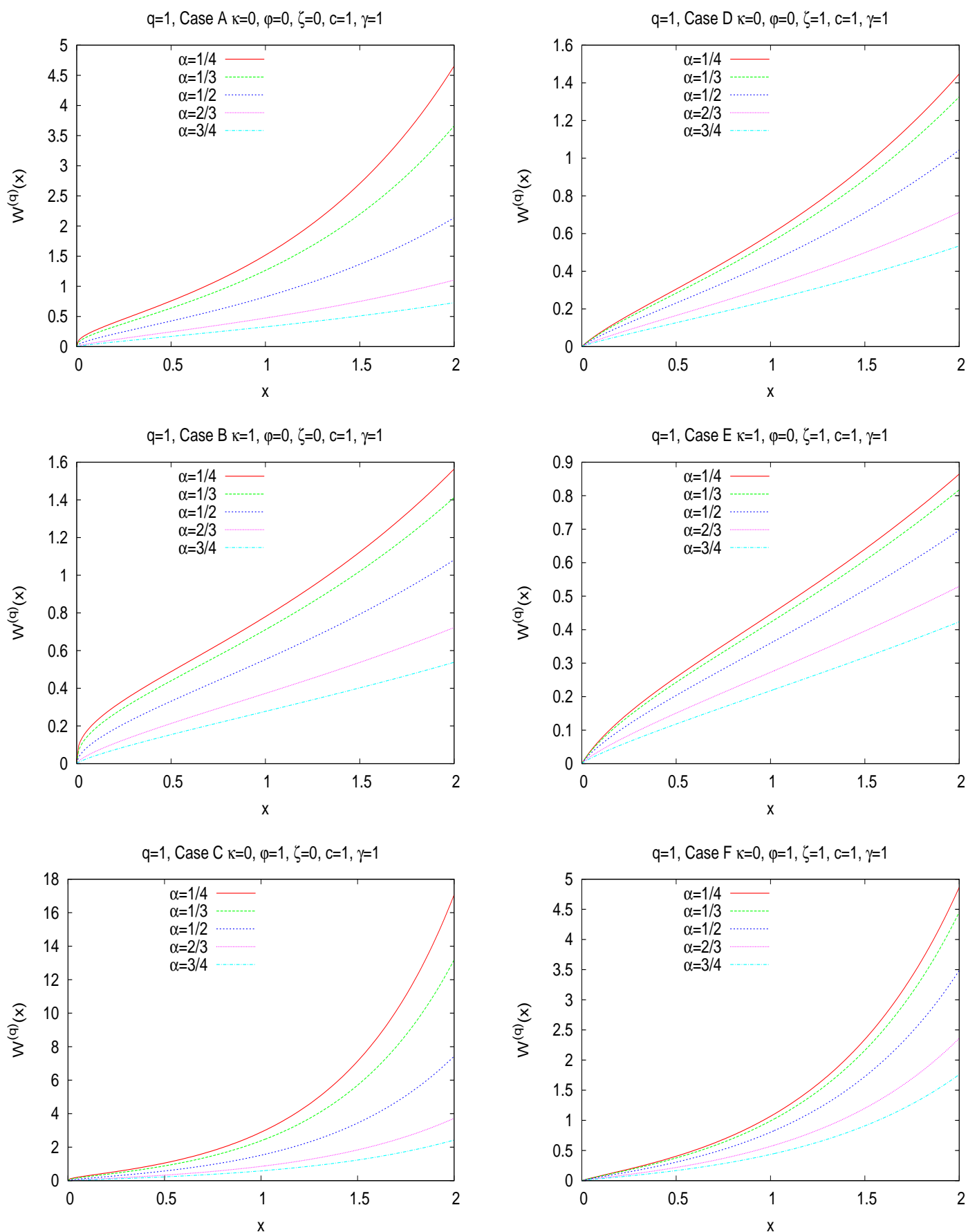

Figure 2: Scale functions $W^{(q)}(x)$ for the GTSC class with $q=1$ and $0<\alpha<1$ 
The key observation is simply, that any Laplace transform, that is of the form $f\left(\theta^{\alpha}\right)$ with $f$ a rational function and $\alpha$ some number, can be inverted in terms of the partial fraction decomposition of $f$ using Mittag-Leffler functions and its derivatives.

In other very recent work, Kyprianou and Rivero (2008) have looked at families of scale functions which are the result of choosing the descending ladder height process to have a Laplace exponent which is a special or complete Bernstein function.

\subsection{Numerical inversion of the Laplace transform}

While our formulas provide closed form expressions for rational $\alpha$ in terms of partial fraction decompositions and Mittag-Leffler functions, those expression can be rather lengthy, involve the zeros of polynomials of high degree, and, experience shows them to be numerically problematic when $\alpha=m / n$ unless $n$ is quite small.

Alternatively one may always consider numerical inversion of the Laplace transform of $W^{(q)}$, which is extremely simple for the GTSC class. Moreover this approach works also for irrational $\alpha$. We use the Bromwich inversion integral for the Laplace transform,

$$
W^{(q)}(x)=\frac{1}{2 \pi i} \int_{r-i \infty}^{r+i \infty} \frac{e^{\theta x} d \theta}{\psi(\theta)-q}
$$

where $r>\Phi(q)$. Let us consider the integrand for $|\theta| \rightarrow \infty$ with $\Re(\theta) \geq r$. The term $e^{\theta x}$ is bounded. If $\zeta>0$ we have $\psi(\theta) \sim \zeta \theta^{2}$, if $\zeta=0$ and $0<\alpha<1$ we have $\psi(\theta) \sim-c \Gamma(-\alpha) \theta^{\alpha+1}$ and $(74)$ is a proper Lebesgue integral. Otherwise it must be interpreted as a principal value integral. Some remarks how to handle this situation and how to improve the numerics and a piece of Mathematica code, that evaluates the scale function by (straightforward, and numerically unsophisticated) integration, are in the extended report. For further material on the numerical evaluation of scale function see also (Surya, 2007, Chapter7, p.111ff).

\subsection{Examples of applications}

Let us briefly conclude by re-enforcing the genuine importance of establishing explicit examples of scale functions from the point of view of modelling by giving three classical examples from the theory of applied probability. It will suffice to consider the role of 0 -scale functions. We shall do so in the light of some of the explicit examples above.

Example 1. Recent literature suggests that a more modern approach to the theory of ruin should involve considering, in place of the classical Cramér-Lundberg process, a spectrally negative Lévy process which drifts to $\infty$ for the risk process. As noted earlier, for any such given Lévy process with Laplace exponent $\psi$, the quantity $1-\psi^{\prime}(0+) W(x)$ is the probability of ruin when the Lévy process is issued from $x$ at time 0 .

Consider the following parent process

$$
X_{t}=(\kappa+\lambda) t-S_{t}^{(1)}-S_{t}^{(2)}, t \geq 0
$$

where $\left\{S_{t}^{(i)}: t \geq 0\right\}, i=1,2$, are subordinators whose Lévy measures, $\nu^{(i)}, i=1,2$, are identified by

$$
\nu^{(1)}(d x)=\lambda \frac{\gamma^{\nu+1}}{\Gamma(\nu)} x^{\nu-1} e^{-\gamma x} d x \quad \text { and } \quad \nu^{(2)}(d x)=\lambda \frac{\gamma^{\nu}}{\Gamma(\nu)}(1-\nu) x^{\nu-2} e^{-\gamma x} d x
$$

for $x>0$.

Following the interpretation of Lévy risk processes in Klüppelberg and Kyprianou (2006) and Huzak et al. (2004) we may think of the parent process as the result of premiums collected at 
rate $\lambda+\kappa$ to offset the result of two competing claim processes. The first has claims arriving at a compound Poisson rate $\lambda$ which are gamma distributed and the second has an infinite intensity of small claims whose aggregate behaviour is similar to that of a stable subordinator with index $1-\nu$. Moreover, these claims are interlaced with larger claims of finite intensity, the tail of whose distribution are similar to those of gamma distributed jumps.

Recalling the discussion in Section 5.2 we have from the second part of Theorem 3 that the probability of ruin from an initial reserve $x>0$ is conveniently given by

$$
1-\frac{1}{\lambda+\kappa}-\frac{\rho \gamma^{\nu}}{\lambda+\kappa} \int_{0}^{x} y^{\nu-1} e^{-\gamma y} E_{\nu, \nu}\left(\rho \gamma^{\nu} y^{\nu}\right) d y
$$

Example 2. If instead we consider the parent process in the previous example reflected in its supremum, then we are looking at the workload of a so called $M / \Pi / 1$ queue (cf Dube et al. (2004)). That is to say, a queue in which work is processed with a constant rate, say $\mu$, and which arrives in packets according to a Poisson point process such that a job of size $x>0$ arrives in the interval $(t, t+d t)$ with probability $\Pi(d x) d t+o(d t)$ where $\int_{0}^{\infty}(1 \wedge x) \Pi(d x)<\infty$. For the case at hand $\mu=\lambda+\kappa$ and $\Pi(d x)=\nu^{(1)}(d x)+\nu^{(2)}(d x)$ and there is again the possibility of interpreting the incoming work as the result of two competing mechanisms. In this case, the stationary distribution of the workload is given by one minus the ruin probability given above.

Example 3. In the very recent work of Loeffen (2007), the classical dividend control problem of De Finetti's dividend problem has been considered in the context of the driving risk process being a general spectrally negative Lévy process denoted as usual $X$. Here the objective is to find the optimal strategy and value to the following control problem

$$
v(x)=\sup _{\pi} \mathbb{E}_{x}\left(\int_{0}^{\sigma^{\pi}} e^{-q t} d L_{t}^{\pi}\right)
$$

where $q>0, \sigma^{\pi}=\inf \left\{t>0: X_{t}-L_{t}^{\pi}<0\right\}$ and $L^{\pi}=\left\{L_{t}^{\pi}: t \geq 0\right\}$ is the process of dividends paid out associated with the strategy $\pi$ and the supremum is taken over all strategies $\pi$ such that $L^{\pi}$ is a non-decreasing, left-continuous adapted process which starts at zero and for which ruin cannot occur by a dividend pay-out.

In Loeffen (2007), the author proves the remarkable fact that, under the assumption that the dual of the underlying Lévy process has a completely monotone density, for each $q>0$, the associated $q$-scale function, $W^{(q)}$, has a first derivative which is strictly convex on $(0, \infty)$. Moreover, the point $a^{*}:=\inf \left\{a \geq 0: W^{(q) \prime}(a) \leq W^{(q)^{\prime}}(x)\right.$ for all $\left.x \geq 0\right\}$ is the level of the barrier which characterizes the optimal strategy. The latter being that dividends are paid out in such a way that the aggregate process has the dynamics of the underlying Lévy process reflected at the barrier $a^{*}$. The value function of this strategy is given by

$$
v(x)= \begin{cases}\frac{W^{(q)}(x)}{W^{(q) \prime}(a)} & \text { for } 0 \leq x \leq a \\ x-a+\frac{W^{(q)}(a)}{W^{(q) \prime}(a)} & \text { for } x>a .\end{cases}
$$

If we are to take any of the parent processes mentioned in this paper as the underlying spectrally negative Lévy process, then it is immediately obvious from (42) that they all have the property that their dual has a Lévy density which is completely monotone. In such a case one may observe the value $a^{*}$ graphically (see for example Figure 2) as well as compute it numerically to a reasonable degree of exactness with the help of software such as Mathematica. 


\section{Acknowledgments}

This work was initiated when both authors were attending the mini-workshop Lévy processes and Related Topics in Modelling in Oberwolfach. We are grateful to the organizers of this workshop and MFO for the opportunity it has provided us. We would also like to thank Ronnie Loeffen for hinting that previously unnoticed scale functions are to be found in the references of Furrer (1998), Asmussen (2000) and Abate and Whitt (1999). Thanks also go to Victor Rivero who commented on earlier versions of this paper as well as an anonymous referee for careful reading of the manuscript.

\section{References}

[1] J. Abate and W. Whitt. Explicit $M / G / 1$ waiting-time distributions for a class of long-taile service-time distributions. Operations Research Letters, 25:25-31, 1999.

[2] L Alili and A.E. Kyprianou. Some remarks on first passage of Lévy processes, the American put and pasting principles. The Annals of Applied Probability, 15(3):2062-2080, 2005.

[3] S. Asmussen. Ruin probabilities. World Scientific Publishing, 2000.

[4] F. Avram, T. Chan, and M. Usabel. On the valuation of constant barrier options under spectrally one-sided exponential Lévy models and Carr's approximation for American puts. Stochastic Processes and their Applications, 100:75-107, 2002.

[5] F. Avram, A.E. Kyprianou, and M. R. Pistorius. Exit problems for spectrally negative Lévy processes and applications to (Canadized) Russian options. The Annals of Applied Probability, 14(1):215-238, 2004.

[6] F. Avram, Z. Palmowski, and M. R. Pistorius. On the optimal dividend problem for a spectrally negative Lévy process. Annals of Applied Probability, 17:156-180, 2007.

[7] R. Bekker, O. Boxma, and O. Kella. Queues with delays in two-state strategies and Lévy input. Journal of Applied Probability, 45:314-332, 2008.

[8] J. Bertoin. Lévy processes, volume 121 of Cambridge Tracts in Mathematics. Cambridge University Press, Cambridge, 1996.

[9] J. Bertoin. Exponential decay and ergodicity of completely asymmetric Lévy processes in a finite interval. The Annals of Applied Probability, 7(1):156-169, 1997.

[10] J. Bertoin, B. Roynette, and M. Yor. Some connections between (sub)critical branching mechanisms and bernstein functions. 2004. arXiv:math/0412322v1 [math.PR].

[11] N. H. Bingham. Continuous branching processes and spectral positivity. Stochastic Processes and their Applications, 4(3):217-242, 1976.

[12] O. J. Boxma and J.W. Cohen. The M/G/1 queue with heavy-tailed service-time distribution. IEEE Journal on Selected Areas in Communications, 16:749-763, 1998.

[13] T. Chan, A.E. Kyprianou, and Savov. M. Smoothness of scale functions for spectrally negative Lévy processes. arXiv:0903.1467v1 [math.PR], 2009.

[14] L. Chaumont. Sur certains processus de Lévy conditionnés à rester positifs. Stochastics and stochastics reports, 47:1-20, 1994. 
[15] L. Chaumont. Conditionings and path decompositions for Lévy processes. Stochastic Processes and their Applications, 64:39-54, 1996.

[16] L. Chaumont and M.E. Caballero. Conditioned stable Lévy processes and the Lamperti representation. Journal of Applied Probability, 43:967-983, 2006.

[17] L. Chaumont, A. E. Kyprianou, and J.C. Pardo. Wiener-Hopf factorization and some explicit identities associated with positive self-similar Markov processes. Stochastic Processes and Their Applications, 119:980-1000, 2007.

[18] S.N. Chiu and C. Yin. Passage times for a spectrally negative Lévy process with applications to risk theory. Bernoulli, 11(3):511-522, 2005.

[19] R. Cont and P. Tankov. Financial modelling with Jump Processes. Chapman \& Hall, CRC Press., 2003.

[20] A.D. Doney and A.E. Kyprianou. Overshoots and undershoots of Lévy processes. The Annals of Applied Probability, 16(1):91-106, 2006.

[21] R. A. Doney. Some excursion calculations for spectrally one-sided Lévy processes. In Séminaire de Probabilités XXXVIII, volume 1857 of Lecture Notes in Math., pages 5-15. Springer, Berlin, 2005a.

[22] R.A. Doney. Hitting probabilities for spectrally positive Lévy processes. Journal of the London Mathematical Society. Second Series, 44(3):566-576, 1991.

[23] R.A. Doney. Fluctuation theory for Lévy processes. In École d'été de probabilités de Saint-Flour, XXXV-2005, volume 1897 of Lecture Notes in Math. Springer, Berlin, 2007.

[24] R.A. Doney. Some excursion calculations for spectrally one-sided Lévy processes. Séminaire de Probabilités, XXXVIII:5-15, 2005b.

[25] P. Dube, F. Guillemin, and R. R. Mazumdar. Scale functions of Lévy processes and busy periods of finite-capacity M/GI/1 queues. Journal of Applied Probability, 41(4):1145-1156, 2004.

[26] D. J. Emery. Exit problem for a spectrally positive process. Advances in Applied Probability, 5: 498-520, 1973. ISSN 0001-8678.

[27] A. Fransén and S. Wrigge. Calculation of the moments and the moment generating function for the reciprocal gamma distribution. Mathematics of Computation, 42(166):601-616, 1984.

[28] H. Furrer. Risk processes perturbed by $\alpha$-stable Lévy motion. Scandinavian. Actuarial Journal, 1:59-74, 1998.

[29] B. Hilberink and L. C. G. Rogers. Optimal capital structure and endogenous default. Finance and Stochastics, 6(2):237-263, 2002.

[30] M. Huzak, M. Perman, H. Šikić, and Z. Vondraček. Ruin probabilities for competing claim processes. Journal of Applied Probability, 41:679-690, 2004.

[31] N. Jacob. Pseudo differential operators and Markov processes. Vol.III. Imperial College Press, London, 2005. Markov processes and applications.

[32] C. Klüppelberg and A.E. Kyprianou. On extreme ruinous behaviour of Lévy insurance risk processes. Journal of Applied Probability, 43:594-598, 2006. 
[33] C. Klüppelberg, A. E. Kyprianou, and R. A. Maller. Ruin probabilities and overshoots for general Lévy insurance risk processes. The Annals of Applied Probability, 14(4):1766-1801, 2004.

[34] V. S. Korolyuk. Boundary problems for a compound Poisson process. Theory Probab. Appl., 19: 1-14, 1974.

[35] V. S. Korolyuk. On ruin problems for a compound Poisson process. Theory Probab. Appl., 20: 374-376, 1975.

[36] S. Kou and H. Wang. First passage times of a jump diffusion process. Advances of Applied Probability, 35:504-531, 2003.

[37] N. Krell. Multifractal spectra and precise rates of decay in homogeneous fragmentations. Stochastic Processes and their Applications 118, 118:897-916, 2007.

[38] A. E. Kyprianou. Introductory lectures on fluctuations of Lévy processes with applications. Universitext. Springer-Verlag, Berlin, 2006.

[39] A.E. Kyprianou and Z. Palmowski. Quasi-stationary distributions for Lévy processes. Bernoulli, 12(4):571-581, 2006.

[40] A.E. Kyprianou and Z. Palmowski. Distributional study of de Finetti's dividend problem for a general Lévy insurance risk process. Journal of Applied Probability, 44(2):428-443, 2007.

[41] A.E. Kyprianou and V. Rivero. Special, conjugate and complete scale functions for spectrally negative Lévy processes. Electronic Journal of Probability, pages 1672-1701, 2008.

[42] A.E. Kyprianou and B. A. Surya. Principles of smooth and continuous fit in the determination of endogenous bankruptcy levels. Finance and Stochastics, 11(1):131-152, 2007.

[43] A.E. Kyprianou, V. Rivero, and R. Song. Smoothness and convexity of scale functions with applications to de Finetti's control problem. To appear in Journal of Theoretical Probability, 2009.

[44] A. Lambert. Completely asymmetric Lévy processes confined in a finite interval. Annales de l'Institut Henri Poincaré. Probabilités et Statistiques, 36(2):251-274, 2000.

[45] A. Lambert. Quasi-stationary distributions and the continuous-state branching process conditioned to be never extinct. Electronic Journal of Probability, 12:420-446, 2007.

[46] R. Loeffen. On optimality of the barrier strategy in de Finetti's dividend problem for spectrally negative Lévy processes. Annals of Applied Probability, 18:1669-1680, 2007.

[47] E. Mordecki and A. Lewis. Wiener-Hopf factorization for Lévy processes having positive jumps with rational transforms. Journal of Applied Probability, 45:118-134, 2005.

[48] M. R. Pistorius. On doubly reflected completely asymmetric Lévy processes. Stochastic Processes and their Applications, 107(1):131-143, 2003.

[49] M. R. Pistorius. On exit and ergodicity of the spectrally one-sided Lévy process reflected at its infimum. Journal of Theoretical Probability, 17(1):183-220, 2004.

[50] M. R. Pistorius. A potential-theoretical review of some exit problems of spectrally negative Lévy processes. In Séminaire de Probabilités XXXVIII, volume 1857 of Lecture Notes in Math., pages 30-41. Springer, Berlin, 2005. 
[51] M. R. Pistorius. An excursion theoretical approach to some boundary crossing problems and the Skorokhod embedding for reflected Lévy processes. Séminaire de Probabilités, 40:287-308, 2007.

[52] J-F. Renaud and X. Zhou. Distribution of the dividend payments in a general Lévy risk model. Journal of Applied Probability, 44(2):420-427, 2007.

[53] L. C. G. Rogers. The two-sided exit problem for spectrally positive Lévy processes. Advances in Applied Probability, 22(2):486-487, 1990.

[54] J. Rosiński. Tempered stable processes. Second MaPhySto Conference on Lévy processes: Theory and Applications, pages 215-220, 2002.

[55] W. Schoutens. Lévy processes in finance. Probability and Statistics. Wiley, 2003.

[56] F. W. Steutel and K. van Harn. Infinite divisibility of probability distributions on the real line, volume 259 of Pure and Applied Mathematics. Marcel Dekker, 2004.

[57] V. N. Suprun. The ruin problem and the resolvent of a killed independent increment process.

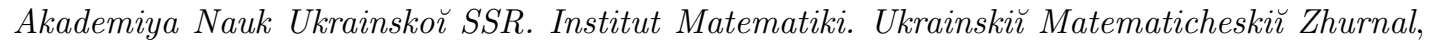
28(1):53-61, 142, 1976.

[58] B. A. Surya. Optimal stopping problems driven by Lévy processes and Pasting Principles. Proefschrift, Utrecht University, 2007. submitted to Journal of Applied Probability.

[59] L. Takács. Combinatorial methods in the theory of stochastic processes. John Wiley \& Sons Inc., New York, 1966.

[60] V. Vigon. Votre Lévy rampe-t-il? Journal of the London Mathematical Society. Second Series, 65(1):243-256, 2002a.

[61] V. Vigon. Simplifiez vos Lévy en titillant la factorisation de Wiener-Hopf. Ph.D. thesis. Laboratoire de Mathématiques de L'INSA de Rouen, 2002b.

[62] V. M. Zolotarev. The moment of first passage of a level and the behaviour at infinity of a class of processes with independent increments. Akademija Nauk SSSR. Teorija Verojatnosteù i ee Primenenija, 9:724-733, 1964. 